
\title{
28 Research Square \\ The Changes of Serum Lipids in Idiopathic Epiretinal Macular Membranes
}

\section{Liguo Feng}

Zhejiang University School of Medicine Sir Run Run Shaw Hospital

\section{Ying Chen}

Zhejiang University School of Medicine Sir Run Run Shaw Hospital

\section{Yesheng Xu}

Zhejiang University School of Medicine Sir Run Run Shaw Hospital

\section{Wenjia Xie}

Zhejiang University School of Medicine Sir Run Run Shaw Hospital

\section{Yi Sun}

Shaoxing Shangyu People's Hospital

\section{Binghong Wang}

Zhejiang University School of Medicine Sir Run Run Shaw Hospital

Yu-Feng Yao ( $\square$ yaoyuf@mail.hz.zj.cn )

Affiliated Sir Run Run Shaw Hospital, Zhejiang University School of Medicine

\section{Research article}

Keywords: idiopathic epiretinal macular membranes, serum lipids, Serum triglycerides, hyperlipemia.

Posted Date: August 14th, 2020

DOI: https://doi.org/10.21203/rs.3.rs-56313/v1

License: (c) (1) This work is licensed under a Creative Commons Attribution 4.0 International License.

Read Full License 


\section{Abstract}

Purpose: To describe the changes of serum lipids in patients with idiopathic epiretinal macular membranes (iERMs).

Methods: A retrospective, observational case control study was performed January 2015 and December 2019 in our unit. A total of 57 subjects with idiopathic epiretinal macular membranes were collected in the study. Senile cataract patients with age matched during hospitalizations were randomly collected as control group. iERMs were diagnosed based on OCT images. Comprehensive ophthalmologic examinations and Systemic examinations were performed. The changes of serum lipids were analyzed.

Results: The iERMs was common in female (71.9\%). Compared to control group, Serum triglycerides were higher in iERMs group $(1.54 \pm 0.66$ vs $1.21 \pm 0.50, P<0.01)$. There was significant difference in BMI with iERM $(23.68 \pm 2.86$ vs $22.50 \pm 2.71, P=0.03)$. After subjects stratified according whether with hypertension diseases, there was still statistical difference in serum triglycerides $(P<0.05)$.

Conclusions: Serum triglycerides and BMI are elevated in patients with iERMs. It suggests that obese people seem to be prone to iERMs. Hyperlipemia seems to play a role in the formation of iERMs.

\section{Background}

As one of the vitreoretinal interface diseases, Idiopathic epiretinal macular membranes (iERMs) or macular pucker are common conditions in older patients. The pathogenesis of epiretinal membranes is still not well elucidated. Various risk factors have been focused on the correlation of iERMs, including ocular and systemic factors, such as myopia, attached cortical vitreous, older age, diabetes and so on [13]. Interestingly, the metabolic abnormalities of serum lipid have been reported in the population with epiretinal macular membranes $[5,6]$.As well known, abnormal lipid metabolism may cause many fibrotic systemic diseases, such as atherosclerosis, non-alcoholic fatty liver disease, macular degeneration, pulmonary alveolar proteinosis and so on [4]. Since the first study to investigate risk factors of ERM was reported, which has demonstrated that serum cholesterol was significantly associated with ERMs [5], there has been a growing body of studies regarding of lipid metabolism in ERM progression $[3,6]$. However, the results were still controversial. In order to further verify the changes of serum lipids in patients with idiopathic macular membranes, we conducted this retrospective case control study.

\section{Methods}

\section{Study population}

This was a retrospective, observational case control study. Subjects were recruited between January 2015 and December 2019 from the Affiliated Sir Run Run Shaw Hospital of Zhejiang University School of Medicine. Vitrectomy combined with membranes peeling was operated in all patients due to idiopathic epiretinal macular membranes. Before surgery, detailed ocular evaluation and comprehensive system 
examination were completed. Eyes with other ocular diseases or surgeries, such as secondary macular pucker, diabetic retinopathy, macular degeneration, uveitis, retinal vessels diseases, infectious eye diseases, cataract surgery, retinal detachment operations, and fundus laser therapy were excluded. The eye with ocular axis length over $25 \mathrm{~mm}$ was also excluded. Autoimmune diseases such as lupus, multiple sclerosis, rheumatoid arthritis, and liver and kidney diseases were excluded. Age-matched senile cataract patients during hospitalizations were randomly collected as a control group.

\section{Ophthalmic examination}

This study was approved by the Ethics Committee of the Affiliated Sir Run Run Shaw Hospital of Zhejiang University School of Medicine. All participants were received comprehensive ophthalmologic examinations, including best-corrected visual acuity (BCVA) with a logarithmic visual acuity chart, noncontact ocular pressure, and slit lamp examination. Lens opacification was graded using a slit lamp by trained ophthalmologists according to lens opacities classification system III. Stereoscopic fundus examination was performed in patients using indirect ophthlmoscope. Fundus photographs were performed in patients with pupillary dilatation by two experienced technicians. Subjects underwent spectral domain-optical coherence tomography (SD-OCT) or optical coherence tomography angiography (OCTA) (Carl Zeiss Meditee, Dublin, Calif., USA) scanning with adequate pupillary dilation. A macular thickness map with six radial 6-mm-long scans was taken, and line scan images with $5 \mathrm{~mm}$ in length through the center of the macula along horizontal and/or vertical axes were acquired. Only images with signal strength of or above $6(\max 10)$ were acquired. Images with large eye movements, disconnecting a large retinal vessel, black bands (caused by blinking) were rejected throughout the examination. OCT scanning was completed by two skilled technicians. OCT parameters were calculated automatically by the equipment's software.

Epiretinal membranes were diagnosed based on OCT images. Specifically, a thin and highly reflective band totally adherent to the neurosensory layer of retina resulted in loss of normal macular outlines was defined as ERM. Also, The ERMs were confirmed by peeling membranes surgery.

\section{Data collection}

Systemic examinations were performed in all patients, including height, body weight, blood pressure, fasting blood glucose, and body mass index (BMI). Cholesterol, high-density lipoprotein (HDL), lowdensity lipoprotein (LDL), triglycerides, homocysteine, serum urea and creatinine were tested by collecting blood samples in all subjects. Hypertension was defined as systolic blood pressure $\geq 140 \mathrm{mmHg}$, diastolic blood pressure $\geq 90 \mathrm{mmHg}$, or being taking antihypertensive drugs. Diabetes mellitus was defined as a fasting blood glucose $\geq 7.0 \mathrm{mmol} / \mathrm{L}, 2$ hour post-meal blood glucose, or being taking hypoglycemic therapy.

\section{Statistical methods}


All statistical analyses were performed using SPSS 22.0. For the association of the variables of ERM, Student's $t$-test was using for the continuous variables and the Pearson $c^{2}$ test was using for the categorical variables. A two-side $P$ value $<0.05$ was considered significantly statistical difference.

\section{Results}

57 subjects with idiopathic ERM were analyzed, included 41 females $(71.9 \%)$ and 16 males $(28.1 \%)$. It was more prevalent in females than males. There were 36 females $(62.1 \%)$ and 22 males $(37.9 \%)$ in the control group. There was not significantly statistical difference in gender ratio between two groups $\left(\chi^{2}\right.$ test, $P=0.36$ ). The average age was $66.8 \pm 8.7$ years ( $49-85$ years) in the ERM group and $72.5 \pm$ 9.3 years (54-90 years) in the control group (Student's $t$-test, $P=0.49)$. There were 23 patients $(40.6 \%)$ with hypertension, included 2 patients with diabetes in ERM group, and 27 patients with hypertension $(46.6 \%)$ in the control group $\left(\chi^{2}\right.$ test, $\left.P=0.69\right)$.

The changes of serum lipids were analyzed between the ERM and control group. The results showed that serum triglycerides were higher in iERMs group than the control group (Table 1). The difference was statistically significant $(1.54 \pm 0.66$ vs $1.21 \pm 0.50 \mathrm{mmol} / \mathrm{L}, P<0.01)$. Also, compared with the control group, there was a statistical difference in BMI with the ERM group $(23.68 \pm 2.86$ vs $22.50 \pm 2.71, P=$ 0.03). There was not significant difference between ERM and control group among the results of cholesterol, HDL, VLDL, LDL, and homocysteine, although it was nearly statistical different in cholesterol levels $(P=0.06)$. 
Table 1

\begin{tabular}{|c|c|c|}
\hline Risk factors & ERM (57) & Control group (58) \\
\hline $\operatorname{Sex}(F, \%)$ & 71.9 & 62.1 \\
\hline Age (years) & $66.8 \pm 8.7$ & $72.5 \pm 9.3$ \\
\hline Serum cholesterol (mmol/L) & $4.83 \pm 0.87$ & $4.63 \pm 0.82$ \\
\hline Serum HDL (mmol/L) & $1.23 \pm 0.29$ & $1.30 \pm 0.32$ \\
\hline Serum VLDL (mmol/L) & $0.74 \pm 0.38$ & $0.62 \pm 0.34$ \\
\hline Serum LDL (mmol/L) & $2.70 \pm 0.73$ & $2.60 \pm 0.65$ \\
\hline Serum TG (mmol/L) & $1.54 \pm 0.66$ & $1.21 \pm 0.50^{\star \star}$ \\
\hline Serum homocysteine $(\mu \mathrm{mol} / \mathrm{L})$ & $12.70 \pm 8.18$ & $15.16 \pm 7.28$ \\
\hline serum urea (mmol/L) & $5.21 \pm 1.12$ & $5.44 \pm 1.27$ \\
\hline Creatinine $(\mu \mathrm{mol} / \mathrm{L})$ & $68.93 \pm 16.46$ & $73.64 \pm 17.59$ \\
\hline BMI $\left(\mathrm{kg} / \mathrm{m}^{2}\right)$ & $23.68 \pm 2.86$ & $22.50 \pm 2.71^{\star}$ \\
\hline Hypertension (\%) & 40.6 & 46.6 \\
\hline \multicolumn{3}{|c|}{$\begin{array}{l}\text { F: female; HDL: high-density lipoprotein; VLDL: very low-density lipoprotein; LDL: low-density } \\
\text { lipoprotein; TG: triglycerides; BMl: body mass index. }\end{array}$} \\
\hline$\star \star P<0.01, * P<0.05$ & & \\
\hline
\end{tabular}

Given that hypertension may be coexisted with hyperlipemia, analyses were stratified according to whether or not with hypertension presented (Table 2). The results showed that it was statistical different in the serum triglycerides whether with hypertension or not, while with hypertension, the results of serum triglycerides were higher in the ERM group than control group $(P<0.01)$. After stratifying, the difference of BMI became not significant between two groups, although the values in BMI with ERM were higher than that of the control group. Interestingly, in patients without hypertension, there was significantly statistical difference in serum urea between ERM group and control group. 
Table 2

\begin{tabular}{|c|c|c|c|c|}
\hline \multirow[t]{2}{*}{ Risk factors } & \multicolumn{2}{|l|}{ hypertension } & \multicolumn{2}{|c|}{ Without hypertension } \\
\hline & ERM (23) & Control group(27) & ERM (34) & Control group(31) \\
\hline Age (years) & $67.91 \pm 8.24$ & $74.70 \pm 8.52^{\star \star}$ & $66.09 \pm 9.11$ & $70.55 \pm 9.56$ \\
\hline Cholesterol (mmol/L) & $4.72 \pm 1.03$ & $4.71 \pm 0.83$ & $4.90 \pm 0.75$ & $4.56 \pm 0.83$ \\
\hline Serum HDL (mmol/L) & $1.18 \pm 0.21$ & $1.30 \pm 0.27$ & $1.27 \pm 0.33$ & $1.29 \pm 0.36$ \\
\hline Serum VLDL (mmol/L) & $0.70 \pm 0.37$ & $0.61 \pm 0.35$ & $0.77 \pm 0.39$ & $0.62 \pm 0.33$ \\
\hline Serum LDL (mmol/L) & $2.65 \pm 0.79$ & $2.70 \pm 0.67$ & $2.73 \pm 0.69$ & $2.50 \pm 0.62$ \\
\hline Serum TG (mmol/L) & $1.47 \pm 0.48$ & $1.17 \pm 0.42^{\star *}$ & $1.57 \pm 0.77$ & $1.24 \pm 0.56^{*}$ \\
\hline Homocysteine $(\mu \mathrm{mol} / \mathrm{L})$ & $13.20 \pm 6.32$ & $14.30 \pm 4.88$ & $12.37 \pm 9.29$ & $15.91 \pm 8.88$ \\
\hline Creatinine $(\mu \mathrm{mol} / \mathrm{L})$ & $73.26 \pm 20.66$ & $75.0 \pm 18.93$ & $66.0 \pm 12.38$ & $72.45 \pm 16.56$ \\
\hline serum urea $(\mathrm{mmol} / \mathrm{L})$ & $5.61 \pm 1.43$ & $5.22 \pm 1.36$ & $4.94 \pm 0.77$ & $5.62 \pm 1.17 \star \star$ \\
\hline BMI $\left(\mathrm{kg} / \mathrm{m}^{2}\right)$ & $24.21 \pm 2.95$ & $23.07 \pm 2.71$ & $23.28 \pm 2.77$ & $22.01 \pm 2.65$ \\
\hline \multicolumn{5}{|c|}{$\begin{array}{l}\text { F: female; HDL: high-density lipoprotein; VLDL: very low-density lipoprotein; LDL: low-density } \\
\text { lipoprotein; TG: triglycerides; BMl: body mass index. }\end{array}$} \\
\hline
\end{tabular}

\section{Discussion}

Idiopathic epiretinal macular membrane is a common vitreo-retinal interface disease, which occurs frequently in old women. In China, series studies have reported that subjects with ERMs were older adults, where female was dominant $[1,6,7]$. Risk factors of ERMs have been investigated in many clinical studies. However, the results were not consistent. In the Hisayama study, Miho et al. found that hypercholesterolemia was significantly associated with ERMs [5]. The Funagata study has shown that HDL cholesterol and fasting plasma glucose were associated with an increased ERM prevalence; however, after adjusting for age and gender, older age and diabetes were the only two risk factors associated with ERM [3]. In another series, Wang and associates found that cardiovascular disease was not associated with ERMs, meanwhile, the prevalence of severe ERMs (preretinal macular fibrosis) was higher in a high cardiovascular risk group than a population-based group. However, the authors did not further analyze the causes of high prevalence of severe ERM in the cardiovascular risk cohort [8]. In the Handan study, the results showed that myopia was associated positively with primary ERM and smoking was an inverse associated factor [1]. In contrast, Ye and colleagues found that besides older age, female and myopia, hyperlipimida were also the risk factors of idiopathic ERM in Jiangning study. However, the authors did not distinguish which compounds were elevated in hyperlipimida [6]. As mentioned above, in our study, hyperlipidemia was significant in the ERMs group. Furthermore, serum triglycerides were higher 
in ERM group than the control group, which was different from previous reports. Although serum total cholesterol was not statistically different between two groups, it was higher in ERMs group $(P=0.06)$. In addition, interestingly, we found that BMI was statistically different between two groups, which was different from previous studies $[5,6]$. In our study, the subjects were those who needed to be vitrectomied for peeling the ERMs. According to previous definition, the ERMs in this study were categorized as preretinal membrane fibrosis (PMF) and not cellophane macular reflex (CMR). The severity of iERMs might be the reason for the different results in this study compared to previous studies.

Given that patients with hypertension might have hyperlipidemia, subjects were further grouped according to whether with hypertension or not. The results showed that Serum TG was significantly different between two groups with hypertension, although the average age of the control group was older than iERMs group. Certainly, in the two groups without hypertension, Serum TG was still statistically different. Our results have suggested that serum TG might play a role in the mechanism of ERMs. Interestingly, in two groups without hypertension, the serum urea was statistically different. However, we had no adequate explanation for the difference.

Our results suggested that serum TG might be a role for the formation of iERMs. The mechanism is still not well known. The presence of remnants of the cortical vitreous following posterior vitreous separation may determine the clinicopathological feature of this entity [9]. As well we known, hyalocytes are mainly distributed close to the retina in the posterior hyaloid and at the vitreous base [10], which has characteristics of tissue macrophages [11]. Using immunocytochemistry and transmission electron microscopy, hyalocytes and vitreous collagen have been observed in the cellular complex of premacular membrane. It is suggested that hyalocytes play a key role for the formation of premacular membrane [12, 13]. The correlation between hyalocytes and serum lipids remains incompletely understood. However, in non-alcoholic fatty liver disease, a fibrous liver disease, the accumulation of lipids in hepatic cells is considered a key event in the development of this disease. Hepatic macropahges and Kupffer cells are thought to play a key role in the progression [4]. As macrophages of posterior hyaloid, it is possible that hyalocytes in the condition of high serum lipids should play a role in the development of iERMs.

There are several limitations in this study, and potential bias should be noted. First, Subjects in this study were retrospectively collected from hospitalization, whose eyes needed to be vitrectomized to peel the epiretinal macular membranes. As mentioned above, epiretinal membranes were categorized as PMF. Subjects with CMF were not included, which might result in selection bias. Second, unlike previous epidemiologic studies which included subjects with ERMs in all ages, the age of populations in this study was older, and we did not stratified for age. Third, we did not investigate the association between OCT characteristics and serum lipids.

In conclusion, we describe the elevation of serum lipid in patients with iERMs. Serum TG and BMI are elevated in the iERMs group. It suggests that obese people seem to be prone to iERMs. Hyperlipemia seems to play a role in the formation of iERMs. Further investigations should be to clarify the etiological relationship between serum lipids and iERMs. 


\section{Declarations}

\section{Ethics approval and consent to participate}

This study was carried out in accordance with the recommendations of Sir Run Run Shaw Hospital, School of Medicine, Zhejiang University in China with informed consent from all subjects. All subjects gave verbal informed consent in accordance with the Declaration of Helsinki. The protocol was approved by Sir Run Run Shaw Hospital, School of Medicine, Zhejiang University.

\section{Consent for publication}

Consent for publication was obtained from the patients.

\section{Availability of data and material}

For datasets in this paper containing clinical data from patients, the authors stated that data would not be shared.

\section{Competing interests}

The Authors declare that there is no conflict of interest.

\section{Funding}

This study has no fund for support.

\section{Authors' contributions}

Design and conduct of the study (LGF, YFY); management, and interpretation of the data (LGF, YCh, YFY); collection, analysis, and preparation (LGF, YS, WJX), and approval of the manuscript (LGF, YCh, YFY, YS, WJX, YSX, BHW).

All authors have read and approved the manuscript.

\section{Acknowledgements}

Design and conduct of the study (LGF, YFY); management, and interpretation of the data (LGF, YCh, YFY); collection, analysis, and preparation (LGF, YS, WJX), and approval of the manuscript (LGF, YCh, YFY, YS, WJX, YSX, BHW).

All authors have read and approved the manuscript.

\section{References}


1. Xin Rong Duan, Yuan Bo Liang, David S. Friedman (2009) Prevalence and Associations of Epiretinal Membranes in a Rural Chinese Adult Population: The Handan Eye Study. IOVS 50(5):2018-23.

2. Todd W. Heilskov, Stephen J. Massicotte, James C.Folk (1996) epiretinal macular membranes in eyes with attached posterior cortical vitreous. Retina 16:279-284.

3. R Kawasaki, JJ Wang, H Sato (2009) Prevalence and associations of epiretinal membranes in an adult Japanese population: the Funagata study. Eye 23, 1045-1051.

4. Anneleen Remmerie, Charlotte L. Scott (2018) Macrophages and lipid metabolism. Cellular immunology 330: 27-42.

5. Miho Miyazaki, Hidetoshi Nakamura, Michiaki Kubo (2003) Prevalence and risk factors for epiretinal membranes in a Japanese population: the Hisayama study. Graefe's Arch Clin Exp Ophthalmol 241:642-646.

6. Hehua Ye, Qi Zhang, Xiaohong Liu (2015) Prevalence and associations of epiretinal membrane in an elderly urban Chinese population in China: the Jiangning Eye Study. Br J Ophthalmol 0:1-4.

7. Wei Xiao, Xiaoyun Chen, William Yan (2017) Prevalence and risk factors of epiretinal membranes: a systematic review and meta-analysis of population based studies. BMJ Open 7:e014644

8. Sarah B Wang, Paul Mitchell, Adam JH Plant (2015) Prevalence and risk factors of epiretinal membrane in a cohort with cardiovascular disease risk, compared with the Blue Mountains Eye Study. Br J Ophthalmol 99:1601-1605.

9. A Gandorfer, M Rohleder, A Kampik (2002) Epiretinal pathology of vitreomacular traction syndrome. Br J Ophthalmol 86:902-909.

10. Hogan MJ, Alvadadr JA, Weddel JE(1971) Histology of the Human Eye. Philadelphia, PA: WB Sanders.

11. Qiao H, Hisatomi T, Sonoda KH (2005) The characterisation of hyalocytes: the origin, phenotype, and turnover. Br J Ophthalmol 89:513-517.

12. Ricarda G. Schumann, Arnd Gandorfer, Jean Ziada (2014) Hyalocytes in idiopathic epiretinal membranes: a correlative light and electron microscopic study. Graefes Arch Clin Exp Ophthalmol 252:1887-1894.

13. Ricarda G. Schumann, Felix Hagenau, Stefanie R. Guenther (2019) Premacular Cell Proliferation Profiles in Tangential Traction Vitreo-maculopathies Suggest a Key Role for Hyalocytes. Ophthalmologica 242(2):106-112. 